\title{
List of Boxes
}

Box 1.1: Craniometric Points $-\mathbf{8}$

Box 4.1: Total IntraVenous Anesthesia (TIVA). - 34

Box 8.1: Can TMS help select patients for Invasive Stimulation? - 85

Box 10.1: Complications of DBS 119

Box 10.2: Possible Mechanisms of Action — 121

Box 10.3: M1 ICS for PD: Tang Guidelines (Canavero 2009) -123

Box 13.1: Overview of Docs 159

Box 13.2: Assessing Consciousness Pre- and Post-treatment - 160

Box 14.1: The DLPFC and Beyond 182

Box 15.1. TINNITUS 187

Box 16.1: NeuroPace ${ }^{\mathrm{TM}}$ System Components - 204

Box 16.2: Technique of implantation (From Smith et al 2009) - 205

Box 18.1: Neuroimaging Studies of Neuropathic Pain - 221

Box 19.1: Electrical field and current density -233

Box 19.2: Procedure for making a 3D realistic head model (Kim et al 2011; Seo et al 2012) — 238 